\title{
SHINING ALONE. ANALIZANDO LA VISIBILIDAD ONLINE COMO ACTIVO DEL SUJETO EMPRESARIAL
}

\author{
Shining Alone. Analysing Online Visibility as an Asset of the \\ Entrepreneurial Subject \\ Javier Zamora García \\ Universidad Autónoma de Madrid \\ javierzg90@gmail.com
}

\begin{abstract}
Resumen:
El presente trabajo investiga bajo qué condiciones es posible afirmar que la web 2.0 está fomentando el desarrollo de subjetividades neoliberales. Para ello, se exploran en primer lugar algunos argumentos encontrados en los Estudios de Internet para sostener esta afirmación. A través de su examen, se propone que, si bien es posible relacionar el diseño de algunas redes sociales digitales con nociones nucleares del neoliberalismo, resulta necesario precisar en qué sentido se desarrolla una relación de poder. En la segunda parte de este artículo, se explica en qué sentido los estudios de neoliberalismo de raíz foucaultiana pueden ofrecer un aparato teórico que resuelva estos problemas. Finalmente, se señalan algunos límites de este modelo, así como futuras líneas de trabajo.
\end{abstract}

\section{Palabras clave:}

Branding personal, neoliberalismo, web 2.0, (Michel) Foucault, subjetivación.

\begin{abstract}
:
The present work explores under which conditions it is possible to claim that the web 2.0 is fostering the development of neoliberal subjectivities. To this end, the first part is devoted to examining some arguments that hold this position in the field of Internet Studies. Based on these arguments it is proposed that, even if it is possible to link the design of some digital social networks with key elements of neoliberalism, it is necessary to pin down the specific sense of a power relation. The second part of this article explains how a Foucauldian Neoliberalism Studies might offer a theoretical apparatus to solve these problems. Finally, some of the limits of this model are suggested, as well as future lines of enquiry.
\end{abstract}

Keywords:

Self-branding, neoliberalism, web 2.0, (Michel) Foucault, subjectivation.

Recibido: 05/11/2018

Aceptado: 15/12/2018 


\section{INTRODUCCIÓN}

Según afirman diversos académicos de los Estudios de Internet, la aparición de redes sociales digitales como Instagram, Youtube, Facebook o Twitter ha democratizado ciertas formas de aparición y representación en el espacio público que antes estaban restringidas a aquellas personas capaces de participar de los medios de comunicación tradicionales, tales como las celebrities, los artistas o los empresarios (Hearn y Schoenhoff, 2016; Marwick, 2015, 2016; Senft, 2013; Turner; 2006). En consecuencia, esta democratización ha provocado que el ciudadano medio sea capaz de proyectar una imagen de sí mismo, cultivar una legión de seguidores y adoptar un perfil público de cara a los demás. Sin embargo, inmersos en una economía de la atención (Marwick, 2016: 343; Senft, 2013: 350), a menudo resulta necesario adoptar una conducta que persiga activamente obtener la mirada ajena. La recurrencia de estos comportamientos ha provocado que diversos autores empleen términos como microcelebrity o Social Media Influencer (SMI) para describir diferentes dimensiones de un mismo fenómeno: la gestión de la identidad online con el objetivo de incrementar su visibilidad, es decir, como si fuera un producto a promocionar $^{1}$.

En el campo de los Estudios de Internet, este tipo de conceptos habitualmente son situados en un contexto social más amplio marcado por dos tendencias paradójicamente coincidentes. Por un lado, como ya hemos avanzado, la web 2.0 y sus múltiples plataformas han diversificado y democratizado las maneras de generar contenido online, desarrollando nuevos modos de darse a conocer sin la necesidad de intermediarios. Por otro, la generación de jóvenes que usa estas herramientas, preparados y ansiosos por obtener o conservar un empleo, con frecuencia se enfrenta a la perspectiva de un mercado de trabajo cada vez más complicado y excluyente. Ante esta situación, a menudo se plantea la hipótesis de que muchos usuarios encuentran en la visibilidad online un potente recurso frente a las cada vez más crudas dinámicas competitivas en el acceso al empleo (Senft, 2013). En efecto, en un contexto marcado por el excedente de información y la saturación de contenido audiovisual, parecería que la capacidad de atraer los ojos de los demás se ha convertido en un marcador de valor tanto social como económico (Zafra, 2015). Además, esta visibilidad es cada vez más importante en campos profesionales como la cultura, la academia y el arte (Zafra, 2017). Por eso, desde enfoques más críticos se ha rechazado limitarse a analizar la búsqueda de atención online como una mera novedad cultural o tecnológica. Así, diversos autores proponen que estas nuevas formas de presentación de la identidad online guardan estrecha relación con la forma

\footnotetext{
${ }^{1}$ Más específicamente, el término microcelebrity engloba una serie de prácticas características de las redes sociales mediante las cuales los usuarios formulan estratégicamente un perfil atractivo, buscan seguidores para confeccionar y cultivar una audiencia, y revelan selectivamente información personal con el objetivo de aumentar su estatus online (Marwick, 2015, 2016; Senft, 2013). Por su parte, el término Social Media Influencer ha sido definido como un nuevo tipo de subjetividad trabajadora que ha surgido en la web 2.0, caracterizada por la forma en que acumula un tipo de capital simbólico similar al que poseen las celebrities. A través de su trabajo en redes sociales, el SMI cultiva tanta atención como sea posible y construye una marca personal con apariencia de autenticidad que puede ser usada posteriormente por compañías privadas para fines publicitarios (Hearn y Schoenhoff, 2016).
} 
en que el espacio digital está siendo construido bajo la influencia de lógicas mercantiles o discursos neoliberales (Hearn, 2017; Iqani y Schroeder, 2016; Senft, 2013). De esta forma, a partir de la penetración de discursos y prácticas provenientes mundo empresarial, las redes sociales digitales estarían facilitando el desarrollo de nuevas formas de subjetividad influidas por el neoliberalismo (Brandinelli y Arvidsson, 2012; Khamis, Ang y Welling, 2017; Lemes de Castro, 2016).

Este artículo pretende interrogarse sobre el alcance de estas afirmaciones desde la óptica del pensamiento político. En un primer apartado se examinarán algunos argumentos ofrecidos por los Estudios de Internet para sostener la influencia del neoliberalismo en la construcción de una subjetividad neoliberal, tales como la influencia del branding personal o las prácticas de algunas empresas dedicadas al posicionamiento online. Como se indicará a continuación, estos argumentos permiten ver que, si bien es posible relacionar de manera general determinadas modalidades de presentación online con el neoliberalismo, resulta mucho más complejo precisar los contornos específicos de dicha relación de poder. A partir de esta problemática, en la segunda parte del texto se planteará una solución a esta falta de precisión teórica recurriendo al aparato teórico de los Estudios de Neoliberalismo que trabajan con una concepción foucaultiana del poder. Así, se sugerirá leer la búsqueda de visibilidad online a partir de la concepción de poder y subjetividad propuesta por Michel Foucault con su curso de 1978-1979 en el Collège de France. En base a los aportes que ofrece esta línea de trabajo, se propondrá que la subjetividad neoliberal hace referencia a la adopción de una estructura identitaria que resulta adaptativa para un contexto social donde los individuos cargan con la mayor parte de los costes de un mercado laboral cada vez más excluyente y competitivo. Como podrá comprobarse, la principal ventaja del modelo foucaultiano es que no propone entender la subjetividad neoliberal como un efecto automático o mecánico del poder. Por el contrario, implica la agencia individual en la creación la propia identidad, así como en la integración dentro de un sistema social del que los individuos dependen para su propio reconocimiento. De esta manera, al tiempo que se aleja de considerar que la agencia humana sea radicalmente libre, el modelo foucaultiano sí que es capaz de integrar los deseos y pasiones del propio individuo, aunque siempre mediados por el sistema social. Finalmente, el modelo de la subjetivación implica una concepción de la relación de poder que nunca se plantea como total, pudiendo así integrar también los usos subversivos y las estrategias de negociación. Para concluir este trabajo, se plantearán en último lugar algunos de los límites de este marco a la hora de interpretar la subjetividad neoliberal en la web 2.0. Por un lado, se sugerirá la necesidad de combinar el análisis foucaultiano de las subjetividades neoliberales con herramientas marxianas que nos permitan dar cuenta de la importancia de la economía política de Internet. Junto a este problema, se señalará asimismo la dificultad que encuentra la teoría del pensador francés para plantear alternativas en el plano colectivo.

\section{LA BÚSQUEDA DE VISIBILIDAD EN LAS REDES SOCIALES DIGITALES COMO PRÁCTICA DE PROMOCIÓN DEL YO: ¿UNA LECTURA TOTALIZANTE?}

Como acabamos de ver, las prácticas de promoción del yo en Internet invitan a plantear una relación entre el diseño de las redes sociales y los discursos neoliberales 
que presentan al individuo como gestor de su propio capital humano. En efecto, para diversos académicos, las redes sociales son plataformas que reflejan -conjuntamente con otros espacios ${ }^{2}$ - la infiltración en la vida social cotidiana de discursos y prácticas provenientes de la nueva cultura empresarial que entiende al individuo al mismo tiempo como una marca y una empresa de sí mismo (Iqani y Schroeder: 2016; Hearn y Schoenhoff, 2016; Page, 2012: 182; Senft, 2013).

De todas ellas, el branding personal (self-branding) que emerge a finales de los 90 es uno de los mejores ejemplos. Dado el eficaz acomodo de sus lógicas en la interfaz de ciertas redes sociales, son diversos los autores que destacan cómo el branding personal ilustra la hibridación entre nuevas formas de presentación identitaria, nociones claves del neoliberalismo -el yo responsable de sí mismo, el mercado como espacio natural de sociabilidad- y una cultura promocional basada en el marketing y la comunicación (Hearn, 2008; Hearn y Schoenhoff, 2016; Khamis, Ang y Welling, 2017; Marwick, 2016). Además de lo anterior, es importante destacar que los nuevos contextos de precariedad laboral están favoreciendo un uso más masivo de lo que anteriormente eran estrategias discursivas destinadas a un grupo reducido de techies y yuppies. Así, si bien los discursos del branding florecieron en la literatura sobre management a finales de los 90 para dar lugar a nuevas formas de creación del valor económico (Hearn, 2008), la idea de transformar la propia identidad en un producto comercial adquiere una nueva dimensión si entendemos que el contexto social en el que se han desarrollado las redes sociales también se caracteriza por la creciente recurrencia de crisis económicas, regímenes de austeridad y precariedad laboral (Hearn y Schoenhoff, 2016).

Ante esta situación, son muchos los autores que destacan cómo el poder comunicativo y las herramientas de gestión del perfil que permiten las plataformas digitales posibilitan la utilización de las técnicas del branding, basadas en la construcción consciente de una metaimagen y metanarrativa de uno mismo con el objetivo de producir valor cultural, y potencialmente, beneficio económico (Brandinelli y Arvidsson, 2012; Hearn, 2008; Khamis, Ang y Welling, 2017). Como anticipara Tom Peters en su famoso artículo The Brand Called You:

the Web makes the case for branding more directly than any packaged good or consumer product ever could. Here's what the Web says: Anyone can have a Web site. And today, because anyone can... anyone does! So how do you know which sites are worth visiting, which sites to bookmark, which sites are worth going to more than once? The answer: branding. The sites you go back to are the sites you trust. They're the sites where the brand name tells you that the visit will be worth your time - again and again. The brand is a promise of the value you'll receive (Peters, 1997).

Tal aproximación a la relación de uno consigo mismo requiere, desde luego, un tipo de dedicación y consistencia que tradicionalmente sólo se veía en figuras como

\footnotetext{
${ }^{2}$ Antes que las redes sociales, la explosión de la telerrealidad (reality shows, talent shows...) ya mostraba cómo determinados formatos televisivos actúan como espacio privilegiado para la activación de aquellos discursos que invitan a construir la identidad como una marca o producto (Hearn, 2008; Khamis, Ang y Welling, 2017; Turner, 2006).
} 
artistas o emprendedores (Senft, 2013: 347). Posteriormente, a través de la difusión del branding personal y otros discursos provenientes de la cultura empresarial, una mayor parte del público pudo acceder a estrategias y métodos que permitían producir una identidad coherente a través de técnicas que invitan a seleccionar determinadas cualidades de nuestra personalidad, empaquetarlas de forma estilizada y articularlas a través de una marca personal (Brandinell y Arvidsson, 2012; Hearn, 2008; Khamis, Ang y Welling, 2017). Pero lo que añaden las redes sociales es que en la actualidad cada vez más personas pueden poner en práctica este tipo de lógicas, en la medida en que las plataformas digitales ofrecen de modo inmediato y accesible la posibilidad de gestionar cómo nos mostramos ante los demás y ante nosotros mismos. Por un lado, la interfaz de las redes sociales nos permite seleccionar aquellos aspectos de nuestra vida personal que queremos exhibir, gestionar su exposición y modelar la forma en que los mostramos, ocultando todo aquello que creamos conveniente. Por otro lado, plataformas como Youtube, Facebook o Twitter facilitan una medición precisa de las interacciones con los otros, principales valedores del yo mercantilizado que se expone en Internet. Así, estas plataformas online permiten cuantificar las interacciones a través de followers, suscriptores o likes (Hearn, 2017: 67). En otras palabras, posibilitan la construcción de audiencias estandarizadas que, convertidas en una especie de moneda, ayudan a evaluar el rendimiento de la marca personal (Hearn y Schoenhoff, 2016).

Como puede deducirse, todos estos comportamientos requieren además un criterio de control de los contenidos que se presentan, orientados siempre a expresar algún aspecto relacionado con la marca personal. Como indicara Peters (1997): "When you're promoting brand You, everything you do - and everything you choose not to do - communicates the value and character of the brand". De este modo, las prácticas de promoción online a menudo se asocian también con una dinámica de constante producción, un modo trabajo siempre activo que acaba provocando lo que para Byung-Chul Han es una cierta compulsión a exhibirnos y autopresentarnos (Han, 2013). Por estas razones, algunos de estos autores han conceptualizado estas formas de producción de identidad como nuevos modos de trabajo inmaterial, intelectual y afectivo que desbordarían las fronteras entre el trabajo y el ocio (Senft, 2013; Hearn y Schoenhoff, 2016; Khamis, Ang y Welling, 2017). De esta manera, serían los propios individuos quienes trabajarían sobre sus propia personalidad, afectos y relaciones con el objeto de amasar visibilidad, produciendo a través de su vida personal y social un valor económico que permite hacer frente a un mercado competitivo y precario (Hearn, 2008; Iqani y Schroeder, 2016; Khamis, Ang y Welling, 2017). Al fin y al cabo, como plantea Alison Hearn, enfrentados a una vida laboral cuya única constante es la presencia de uno mismo, el propio yo es lo único que puede otorgar una coherencia y un carácter distintivo capaces de atraer la atención de inversores o empleadores (Hearn, 2017: 65). De este modo, el resultado de este proceso es que los individuos acabarían siendo al mismo tiempo tanto trabajadores de sí mismos como también una marca o producto a promocionar en el mercado.

Es precisamente esta actitud consciente de continua gestión de la marca personal lo que está sugiriendo un lucrativo nicho de mercado para algunas compañías privadas dedicadas al posicionamiento online. Así, la competición por la visibilidad ofrece la oportunidad de que los paquetes de metadatos generados por los usuarios, 
tradicionalmente vendidos a compañías de marketing o publicidad (Arvidsson y Colleoni, 2012; Srnicek, 2017), obtengan también otros usos. El ejemplo más claro lo encontramos en los servicios ofrecidos por compañías como Klout. En primer lugar, Klout ofrece una medida objetiva del nivel de "influencia" de los usuarios en la red a partir de una escala 1-100, reflejando su "impacto" a través de las diferentes redes sociales. Sin embargo, lejos de limitarse a ello, la empresa también aporta guías y consejos para aumentar la puntuación. Finalmente, proporciona determinadas ventajas como el acceso privilegiado a ciertos bienes o servicios para aquellos usuarios más "influyentes". Ante estas circunstancias, investigadoras como Alison Hearn han destacado que las nuevas economías digitales no solo implican la extracción de valor a partir del contenido que generan los usuarios, sino también un modelado de sus patrones de conducta en la red e incluso fuera de ella, pues, como afirma la investigadora canadiense, la puntuación en Klout comienza también a ser utilizada en procesos de selección de empleo (Hearn, 2017).

A la luz de estos análisis, es indudable que el trabajo realizado por los Estudios de Internet revela una conexión crucial entre la arquitectura de las redes sociales y determinadas lógicas propias del neoliberalismo. En primer lugar, la descripción del yo como una empresa o marca humana aparece como un elemento que articula toda la promoción de la identidad online a través de técnicas como el branding o el uso de los servicios de empresas como Klout. Por otro lado, la caracterización del neoliberalismo como un marco simbólico que promueve el mercado como forma de relación refleja a nivel discursivo la materialidad de ciertas dinámicas sociales que vertebran la vida social online, basadas en la competición, la seducción y la actualización del valor propio. Sin embargo, y a pesar de la aparente presencia de estos elementos, la mayoría de los trabajos de los Estudios de Internet no profundizan lo suficiente en caracterizar cómo influyen lógicas neoliberales sobre el comportamiento individual. ¿Qué relación concreta se da entre los discursos neoliberales, las economías de mercado y las prácticas cotidianas de aquellos usuarios que habitan las redes sociales? ¿Penetra el neoliberalismo la totalidad de la web 2.0., o son posibles otros usos? Por otro lado, ¿modela la arquitectura de las redes sociales los deseos de los individuos hasta el punto de hacerles desear la visibilidad, o más bien canaliza unas intenciones que ya tenían estos previamente? ¿Qué estímulos recibe el individuo para dejarse penetrar en su vida personal por las estrategias de branding o para contratar servicios de empresas como Klout? En definitiva, ¿en qué sentido puede hablarse de relaciones o estructuras de poder que produzcan una subjetividad neoliberal?

A mi modo de ver, todas estas cuestiones resultan fundamentales para evitar ciertos problemas, a menudo relacionados con una caracterización deficiente o simplista de lo que entendemos por neoliberalismo y subjetividad neoliberal. De no responderlas adecuadamente, el estudio de estos fenómenos corre el riesgo de reproducir viejas y nuevas limitaciones que impiden comprenderlos en toda su complejidad. Así, por ejemplo, uno de los problemas presentes en algunos trabajos es que en ocasiones se privilegia en exceso la importancia de procesos económicos hasta el punto de ignorar el peso de otro tipo de dinámicas sociales (Zajc, 2015). Así, describir la sobreexposición del yo como un proceso de mera acumulación de capital social, cultural o simbólico (Fuchs, 2013) oscurece matices que son claves para comprender la 
evidente implicación de dinámicas de reconocimiento en el cultivo de una reputación online. Al fin y al cabo, no puede olvidarse que la visibilidad no solo implica un recurso monetizable, sino que se trata de un fenómeno igualmente relacionado con la existencia social de los individuos.

Por otro lado, sobreestimar la importancia de determinadas lógicas de poder también impide capturar la complejidad del espacio de la web 2.0, donde conviven fenómenos a menudo contradictorios. Como señalan diversos autores (Chouliaraki, 2010; Conrad Murray, 2015; Iqani y Schroeder, 2016; Tiidenberg y Cruz, 2015), junto a prácticas que parecen reificar nuestra identidad convirtiéndola en un producto, la naturaleza relativamente abierta y participativa de redes sociales como Facebook, Twitter, Youtube o Instagram también permite la aparición de formas de presentación del yo que subvierten o disputan ideales normativos. En efecto, tal y como muestran estos trabajos, las redes sociales son también un espacio en el que se desarrollan formas de aparición pública que abren el debate sobre problemáticas sociales contemporáneas (modelos de belleza, desórdenes alimenticios, rechazo al envejecimiento...). De esta manera, parecería que concentrarse exclusivamente en la influencia de determinados discursos ideológicos podría impedir comprender la ambivalencia del espacio digital, donde también se desarrollan contranarrativas que tratan de hacer hueco a nuevas identidades, por mucho que estas formas de acción también se encuentren expuestas a ser apropiadas por dinámicas de mercado (Chouliaraki, 2010).

Finalmente, ciertas precauciones también resultan importantes ante el uso totalizante de categorías que dificultan calibrar el grado de agencia individual en contextos como éste. Así, como indica Melita Zajc (2015), un uso excesivo de la categoría del trabajo para describir la actividad en las redes puede conducir a considerar la mercantilización de toda actividad en la web 2.0, tal y como parecen sugerir algunos trabajos de Christian Fuchs (2010; 2013; Fuchs y Sandoval, 2014; Fuchs y Sevignani, 2013). Por otro lado, una concepción mecanicista del funcionamiento del poder blando puede llegar a eliminar por completo el papel de la agencia y los deseos individuales, haciendo parecer que el neoliberalismo actúa como una entidad en autoexpansión que absorbe toda la actividad de los usuarios.

Por todas estas razones, parecería que no basta con vincular las prácticas de búsqueda de visibilidad con discursos neoliberales, sino que resulta necesario profundizar en la naturaleza de esta relación. ¿Cómo explicar, si asumimos la penetración de la red por discursos ideológicos, la existencia de usos subversivos y prácticas de resistencia? ¿Hasta qué punto la web 2.0 se articula dentro de un sistema social inescapable atravesado por dinámicas de promoción del yo? ¿Cómo encajar ese paisaje con el entusiasmo, la pasión y en ocasiones la clara conciencia con la que los individuos adoptan determinados comportamientos que convierten su propia identidad en una mercancía? En mi opinión, todas estas preguntas requieren una reflexión más profunda sobre qué entendemos por subjetividad neoliberal, y en qué sentido esta forma de poder coacciona o no la libertad de los individuos. En la próxima sección, intentaré explicar cómo un marco teórico basado en los Estudios de Neoliberalismo que continúan el trabajo de Michel Foucault puede resultar útil para pensar estas cuestiones. 


\section{SUBJETIVIDADES NEOLIBERALES, SITUACIONES DE MERCADO Y ESTRATEGIAS DE SUPERVIVENCIA}

Sin lugar a dudas, una de las contribuciones más importantes al estudio del neoliberalismo y el tipo de subjetividad que produce reside en la línea de investigación iniciada a partir de uno de los cursos de Michel Foucault en el Collège de France titulado Nacimiento de la biopolítica. A pesar de su muerte, la propuesta de análisis sobre el neoliberalismo que ofrece Foucault ha tenido un largo recorrido, como muestra el trabajo de distintos académicos contemporáneos a partir del utillaje teórico propuesto por el autor francés (Brown, 2015; Feher, 2009; Lazzarato, 2013; Laval y Dardot, 2013; López Álvarez, 2016). Para el presente artículo, creo interesante rescatar dos aportaciones de este enfoque.

La primera de las grandes virtudes de la propuesta foucaultiana es que no plantea comprender el neoliberalismo como una ideología, sino como una razón gubernamental (Foucault, 2009: 24-27), o, como se describía en El sujeto y el poder, una racionalidad política (Foucault, 1998: 4) ${ }^{3}$. A juicio de Wendy Brown, esta concepción nos permite entender que el neoliberalismo no solo implica un conjunto de políticas económicas o una serie de discursos legitimadores, sino más bien una visión del mundo estructurada alrededor de un orden normativo de la razón (Brown, 2015: 60). En otras palabras, una cosmovisión que integra y da coherencia a un sistema social y que, por tanto, se estructura sobre la base de unos ideales normativos. Una visión del poder que, como indica Brown, resulta particularmente interesante para comprender la forma en que actúa el neoliberalismo en sociedades euroatlánticas, en las que este conjunto de ideas "se ha enraizado de modo más profundo en los sujetos y en el lenguaje, en las prácticas ordinarias y en la conciencia" (Brown, 2015: 57).

En el caso de la racionalidad neoliberal, la carga normativa que estructura al sujeto se encontraría relacionada con la extensión de lenguajes, prácticas y lógicas de mercado al resto de la sociedad, en la medida en que es el mercado el que sirve de

\footnotetext{
${ }^{3}$ Es importante tener en cuenta que la noción de racionalidad presenta ciertas diferencias con el uso tradicional del concepto de ideología más asociado a determinados enfoques marxistas. Como señala Mauro Benente, a pesar de las pocas referencias en la obra de Michel Foucault al término ideología, es posible entender que para el autor francés dicho término resultaba demasiado restrictivo, en la medida en que el marxismo lo había utilizado para referirse únicamente a discursos políticamente interesados que sostienen estructuras de poder, ocultando el acceso a un saber verdadero. Para Foucault, por tanto, el concepto de ideología tradicionalmente había cargado con connotaciones negativas, vinculadas a un cierto falseamiento en el acceso a la verdad y que acababan produciendo una separación entre ideología y ciencia. Por el contrario, el filósofo francés consideraba que la verdad no reside en universales, sino que siempre depende de sus condiciones de emergencia o aceptabilidad en sociedad, es decir, de su episteme (Benente, 2015). A mi juicio, esta posición evidencia en qué medida su concepto de racionalidad combina una visión más histórica de la verdad con la existencia de estructuras de poder dentro de las cuales ésta emergería. No obstante, a pesar de las disputas de Foucault con el marxismo de su época, considero que es posible tender puentes entre el término de racionalidad y aquellas nociones de ideología más apoyadas en la sociología y la antropología, como la que sostiene Ėve Chiapello (2003) a partir de los trabajos de Paul Ricoeur y Karl Mannheim, o la propuesta por Michael Freeden (1996).
} 
principio, forma y modelo para el Estado y la sociedad (77). Tanto para liberales como para neoliberales, la noción de mercado ofrece el modelo por excelencia para vehicular la sociabilidad, puesto que facilita la determinación del valor efectivo de las cosas a través de la formación de precios (Foucault, 2009: 129). No obstante, Foucault precisa que, a pesar de coincidir en la valoración positiva de la forma mercado, es necesario especificar ciertas diferencias entre la racionalidad de gobierno liberal y la neoliberal. De todas ellas, aquí cabe destacar principalmente dos: en primer lugar, para el neoliberalismo el principio regulador de la sociedad y la dinámica social por excelencia ya no es el intercambio, sino la competencia, dado que es la dinámica competitiva la que mejor asegura la formación de precios (130 - 131). En segundo lugar, para los neoliberales el mercado ya no es una realidad natural que, debidamente protegida, permita un funcionamiento armonioso de la sociedad. Por el contrario, el neoliberalismo considera que el mercado se asemeja más a un tipo ideal que debe ser continuamente producido $(133,138)$.

A la hora de extender el modelo del mercado a la totalidad del campo social, una de las estrategias más importantes del neoliberalismo pasa por la generalización de una racionalidad económica, que en plano individual se manifiesta en la articulación de un cierto tipo antropológico. En concreto, este tipo antropológico consistiría en un modelo particular de homo oeconomicus simbolizado por forma empresa ${ }^{4}$. Para el neoliberalismo, la empresa constituye una ficción reguladora individual que permite compatibilizar, en un sentido ciertamente platónico, las virtudes de la ciudad con las virtudes del hombre (Brown, 2015: 20). Así, tal y como la sociedad debe verse como un entramado de empresas o "una suerte de empresa permanente y múltiple" (Foucault, 2009: 239), el sujeto es también concebido como empresario de sí mismo, dueño de su propio capital y su propio producto (228).

Esto no quiere decir, como señala el propio Foucault, que abordar al individuo como empresa implique la asimilación inmediata de cualquier comportamiento a uno económico (252), como si el neoliberalismo fuera una sustancia que lo recubre todo. Más bien, la propuesta del autor francés pasa por comprender que la forma que tiene el individuo de constituirse está mediada por el contexto sociopolítico que habita, en el que ciertas formas de articulación del yo resultan hegemónicas. En palabras de Butler, "ciertos códigos prescriptivos históricamente establecidos determina[n] un tipo particular de formación del sujeto" (Butler, 2009: 30). En este sentido, para el neoliberalismo es la ficción regulativa del yo-empresa la que actúa como una clave de inteligibilidad que, en determinados ambientes, es propuesta para analizar el

\footnotetext{
${ }^{4}$ Como señala Brown, el concepto de homo oeconomicus ha sufrido diversas transformaciones desde sus primeras formulaciones en Smith, en el que era visto como una criatura de mercado propensa al cambio, la permuta y el trueque (Brown, 2015: 122; original en Smith, 1958: 19). Aunque Foucault registra la evolución de esta figura, ese trabajo de adaptación histórica ha continuado también tras su muerte. Así, con el proceso de financiarización de la economía, autores como Michel Feher o Wendy Brown consideran que el modelo de homo oeconomicus bajo condiciones actuales es cada vez más el del capital de financiero o de inversión, en la medida en que el objetivo de este capital es más la apreciación de su valor de mercado que la maximización del beneficio económico de manera inmediata (Feher, 2009: 27; Brown, 2015: 41).
} 
comportamiento de los individuos. En efecto, gracias al desarrollo de teorías como la del capital humano o la elección racional, esta manera de comprender al individuo articula un cierto tipo de racionalidad que permite reinterpretar en clave económica las relaciones de los individuos consigo mismos, con el tiempo, y con el entorno (Foucault, 2009: 241). Así, por ejemplo, mientras que la figura clásica del trabajador asalariado solo vendía su fuerza de trabajo, el nuevo modelo de homo oeconomicus neoliberal permite desdibujar la división entre vida y trabajo, de modo que toda actividad personal -incluidas las relaciones o los pasatiempos- puede ser evaluada en relación a su capacidad de apreciar el valor del individuo (Feher, 2009). De esta manera, la propia relación del individuo consigo mismo cambia: al gestionar la empresa personal un capital humano, la apreciación del propio valor de mercado aparece como un objetivo que estructura la propia vida (27). Por otro lado, las relaciones con los otros también sufren una transformación bajo la óptica del yo empresarial, pues estos otros pueden ser vistos como competidores o como recursos e inversiones que deben ser administrados individualmente bajo la forma de capital social (Brown, 2015: 44 - 45). Finalmente, a partir de esta nueva forma de interpelar a los individuos en tanto que capital, se producen también determinadas resignificaciones de espacios anteriormente no mercantilizados: la educación o la salud comienzan a ser vistas como una inversión a futuro, del mismo modo que la vida amorosa puede abordarse desde el punto de vista del emprendimiento (Brown, 2015: 36). Como señala la autora estadounidense, "ya sea a través de los 'seguidores', likes y retweets de los medios sociales, ya sea a través de clasificaciones y calificaciones de cada actividad y esfera, ya sea de modo más directo a través de prácticas monetizadas", el modelo del homo oeconomicus neoliberal promueve que el individuo configure todos los aspectos de su vida "como decisiones y prácticas estratégicas relacionadas con mejorar el valor futuro de uno mismo" (41).

A la luz de estos argumentos, puede comenzar a vislumbrarse en qué sentido las redes sociales actúan como sostén material para esta ficción del yo que es la empresa. $\mathrm{Si}$ el individuo se entiende a sí mismo metafóricamente como una empresa en el mercado, debe asumir la responsabilidad de promocionarse y darse a conocer. Como ya se ha visto, serían entonces las nuevas plataformas digitales las que facilitarían el acceso a esa visibilidad. Sin embargo, esto no acaba de explicar por qué el homo oeconomicus neoliberal resulta una ficción atractiva para los usuarios de las redes sociales, y de modo más general, para los ciudadanos de las democracias occidentales. Para comprender mejor este aspecto, conviene destacar otra de las virtudes de la propuesta de análisis foucaultiano del neoliberalismo. En este caso, esta virtud está vinculada con la forma en que Michel Foucault conceptualizó las relaciones entre el poder y el sujeto durante sus últimos años de vida.

Para el autor francés, el poder en las sociedades contemporáneas se entiende como un modo de acción que actúa sobre las acciones, una estructuración del campo de acción del otro que necesariamente exige la libertad del individuo sobre el que actúa para poder funcionar (Foucault, 1998: 15). En este sentido, una de las contribuciones más interesantes de Foucault pasa por sugerir que la eficacia del neoliberalismo como estructura de sentido no reside principalmente ni en su capacidad de coacción ni en su persuasión a través de la proliferación de mensajes ideológicos. Por el contrario, la fuerza de la relación de poder que desarrolla el neoliberalismo reside en su capacidad 
para la creación de una serie de ambientes donde la mejor conducta es la conducta neoliberal. En otras palabras, es interpelando al individuo como un inversor o competidor en áreas como la salud o la educación como se fomenta la reinterpretación de fenómenos cotidianos de forma coherente con una racionalidad económica de mercado (Brown, 2015: 44; Foucault, 2009: 261). Bajo este enfoque, el yo empresarial ya no sería un modelo abstracto -una concepción del individuo característica de determinadas teorías políticas-, sino algo diferente: una estrategia de supervivencia concreta que, a través de una serie de discursos o prácticas materiales, se ofrece como la mejor conducta para una serie de situaciones que exigen la asunción privada de una serie de riesgos y responsabilidades.

Teniendo en cuenta estas aportaciones -el neoliberalismo como racionalidad que se encarna en el homo oeconomicus, y la eficacia de una relación de poder basada en la producción de ambientes-, podemos ya comprender mejor la naturaleza específica del vínculo de poder que une al neoliberalismo con la arquitectura de las redes sociales.

En este sentido, la primera ventaja de esta interpretación es que se aleja de una concepción mecanicista del poder que plantee que toda aparición del yo en la web 2.0 implica automáticamente una mercantilización de uno mismo. Por el contrario, lo que se revela a través de esta lectura es que el sujeto neoliberal no se produce de forma automática por el influjo de ideologías, sino que, como insiste Butler, todo sujeto es siempre contribuyente activo de su propia subjetivación (Butler, 2001b; 2009). Así, podríamos proponer que la infiltración de la normatividad neoliberal circula menos a través de una especie de contagio vírico que a través de estrategias de adaptación utilizadas por individuos que tratan de sobrevivir en sistemas sociales potencialmente excluyentes. Lejos, por tanto, de entender que los modos de subjetivación producen al sujeto como un efecto necesario de sus contenidos, la agencia se revela como elemento clave en la adopción de aquellos modelos antropológicos que mejor se integran en un sistema social. No obstante, como señala también Butler, se trata de una agencia limitada, pues ni está del todo determinada ni es radicalmente libre, sino que está constreñida siempre al marco limitado dentro del que se desarrolla (33).

Es precisamente esa visión de una agencia limitada la que nos permite comprender mejor la sensación de inescapabilidad que sugieren algunos trabajos de los Estudios de Internet cuando describen la emergencia de un sujeto neoliberal en la red. En este sentido, esta inescapabilidad no se relacionaría tanto con la existencia de un poder omniabarcante, sino con la forma en que el quehacer del propio individuo se encuentra restringido en dos sentidos distintos, referidos a continuación:

A nivel material, nuestras acciones están limitadas por los ambientes de mercado que exigen la asunción individual de una serie de responsabilidades. Para entender el alcance de esta cuestión, conviene que recordar que, como colofón a décadas de privatización de las protecciones sociales e individualización de la política social, la crisis de 2008 significó un incremento significativo en la responsabilización del individuo de su propia seguridad (Lazzarato, 2013: 59). De este modo, la deflación salarial, las medidas de austeridad y el crecimiento de la deuda han contribuido a intensificar una situación generalizada de precariedad vital, en la que, además, se 
impone una asunción subjetiva de los costos y riesgos que han sido progresivamente externalizados por el Estado y por las empresas (Brown, 2015; Lazzarato, 2013; Feher, 2009). Por tanto, la ficción del individuo como capital resulta funcional para una situación en que son los ciudadanos individuales los que tienen que asumir cada vez más peso gestionando su propia empleabilidad, su descenso salarial o la reducción de los servicios sociales. Finalmente, la ficción reguladora de la empresa también resulta ventajosa para Estados y empresas, que externalizan sus costos y recortan en protección social. Así, no ser una marca a menudo entra en conflicto con escenarios que exigen serlo para sobrevivir. De lo contrario, uno se enfrenta al riesgo de fracasar económicamente.

Por otro lado, la agencia individual también estaría condicionada a nivel psicosocial, pues la identidad de los individuos depende de su capacidad para adaptarse a ciertos marcos de reconocimiento. En este sentido, es importante recordar que los procesos de subjetivación, para Foucault, siempre implican 1) la subordinación a un tipo de identidad particular, como en este caso puede ser el yo empresa; pero también 2) la posibilidad de existir socialmente bajo unos determinados marcos de sentido (Foucault, 1998). En palabras de Butler, si el individuo está obligado a "buscar el reconocimiento de su propia existencia en categorías, términos y nombres que no ha creado", entonces "las categorías sociales conllevan simultáneamente subordinación y existencia" (Butler, 2001b: 31). Por eso, la influencia de ciertas ficciones hegemónicas sobre el sujeto no puede entenderse como un "por encima", una estructura suplementaria que pueda eliminarse para desvelar una subjetividad auténtica (Foucault, 1998: 17). Por el contrario, el poder es algo que "también forma al sujeto, que le proporciona la misma condición de su existencia y la trayectoria de su deseo" (Butler, 2001b: 12). De este modo, el poder actúa no solo como influencia externa, sino también como elemento de integración social y fijación identitaria, en la medida en que, como animal social, el ser humano se encuentra inmerso en una serie de prácticas reguladoras que mantienen la coherencia y la continuidad de la persona, asegurando también la propia inteligibilidad social (Butler, 2007: 71). Por esta razón, poner en cuestión esas normas, desertar de esos modos de presentación, implica poner en riesgo las propias condiciones de reconocibilidad de la propia identidad (Butler, 2009: 38). En otras palabras, implica correr el riesgo de aparecer socialmente bajo formas no del todo comprensibles por los otros. Una conducta que retrata con bastante precisión el riesgo que corren aquellos que desertan o fracasan en la promoción de su propia marca, expuestos no solo al riesgo del desempleo, sino también al costo de encarnar una identidad perdedora que resulta penalizada por las normas sociales.

Finalmente, esta concepción de la relación de poder neoliberal también permite integrar la existencia de acciones subversivas. Así, en la medida en que el poder ya no se entiende como capaz de sujetar la totalidad del individuo, se puede comprender en qué medida los individuos, gracias a su reflexividad, pueden negociar o discutir los marcos de subjetivación dentro de los que se desarrollan (Butler, 2001a). A este respecto, esta perspectiva permite explicar en qué sentido las redes sociales, a pesar de su disposición para la promoción del yo, pueden servir también para usos subversivos, tales como los recogidos al principio de este artículo. Algo especialmente 
claro en la medida en que la mayoría de estos usos subversivos no cuestionan la economía de la visibilidad, sino que tratan de instrumentalizarla con fines diversos.

\section{CONCLUSIONES}

A lo largo de este artículo he tratado de examinar hasta qué punto podemos afirmar que las redes sociales están contribuyendo a producir una subjetividad neoliberal. Así, he tratado de ofrecer una explicación para la búsqueda de la atención online que permita capturar adecuadamente el funcionamiento de relaciones de poder vinculadas con el neoliberalismo, alejándome de aquellas concepciones mecanicistas o totalizantes. Para ello, he sugerido interpretar la búsqueda de la visibilidad online desde el marco de los Estudios de Neoliberalismo de raíz foucaultiana, proponiendo interpretar este fenómeno como parte de una estrategia más amplia de supervivencia en contextos de precariedad. En estos contextos, el individuo -socialmente aislado y económicamente precario- encuentra en la visibilidad online no solo una confirmación de su existencia social, sino una inversión de cara a futuribles empleos. Siendo así, la visibilidad se presenta como un recurso esencial para la promoción de un yo que se interpreta a sí mismo como una empresa o un capital humano. En este sentido, las redes sociales digitales formarían parte a su vez de un dispositivo de subjetivación neoliberal por su capacidad de facilitar el acceso a la visibilidad.

Como acabamos de ver, es evidente que el marco ofrecido por Foucault en su propuesta del Collège de France puede ayudarnos a articular un concepto más útil para aquello que algunos autores denominan neoliberalismo. De manera general, el concepto de racionalidad neoliberal sugerido por Foucault ofrece una matriz teórica que ilumina importantes aspectos de las relaciones entre subjetividad y poder. Así, a la hora de comprender esta aproximación, es importante destacar que la concepción foucaultiana del sujeto no solo implica las formas en que el poder condiciona las acciones de los individuos, sino que también se refiere a la necesidad de estos individuos de integrarse y ser reconocidos dentro de los marcos simbólicos de un sistema social marcado por la existencia de ambientes de mercado.

Asumir, sin embargo, que la subjetividad neoliberal tiene su origen en la formación de ambientes de mercado, reconociendo la relación entre la conciencia y sus condiciones materiales, implica una importante consecuencia. Y es que, a pesar de la disputa de Michel Foucault con el marxismo, es esta corriente teórica la que mayor atención ha prestado a las economías de mercado, dentro de las cuales se desarrolla en gran medida el modo de producción capitalista. Además, como bien indica Srnicek (2017), la web 2.0 es el producto de compañías privadas como Facebook, Youtube o Twitter que, si bien funcionan en un mundo simbólico, son antes que nada compañías privadas dentro de una economía capitalista. En efecto, responden a lógicas propias de este sistema económico: competencia, tendencia al monopolio, búsqueda irrefrenable del crecimiento, etc. Finalmente, en el análisis de los discursos, no puede perderse de vista la cada vez más estrecha relación entre la creación cultural y la economía, diagnosticada a lo largo del último siglo por numerosos autores (Debord, 1999; Wernick, 1999; Read, 2003). Por todas estas razones, en el análisis de las subjetividades neoliberales dentro de la web 2.0, probablemente entonces convenga combinar el aparato foucaultiano para el análisis de la producción de la subjetividad 
con aquellas herramientas marxianas que nos permiten analizar el modo de producción dominante.

Finalmente, se ha sugerido también que la búsqueda de visibilidad en las redes sociales adquiere mayor sentido si la entendemos como el producto de un hacer que está limitado o restringido a nivel material y social por una serie de marcos materiales y sociales. Sin embargo, como también se ha visto, el concepto de subjetivación implica también que esos marcos pueden subvertirse, resistirse o negociarse. Por tanto, esta interpretación soluciona otro de los problemas mencionados en este artículo, ya que no niega la posibilidad de acciones subversivas individuales en el espacio digital. Sin embargo, el modelo foucaultiano presenta al mismo tiempo dificultades a la hora de teorizar la construcción de alternativas en el plano colectivo. En este sentido, el propio trabajo de Foucault es prueba de este límite, en la medida en que su trayectoria demuestra un interés mucho más claro por cuestiones éticas y subjetivas que por la elaboración de otras formas de gubernamentalidad. Una cuestión que no deja de ser paradójica, pues es el propio Foucault quien, señalando que no es posible pensar al yo más allá del poder, nos desafía a imaginar qué otros modos de subjetivación son posibles, qué otras gubernamentalidades pueden suponer una alternativa a la que aquí analizamos ${ }^{5}$. No obstante, y a pesar de notables intentos desde coordenadas teóricas cercanas a la teoría del populismo (Cadahia y Blengino, 2018), parece más fácil seguir a Mario Domínguez (2018) cuando afirma que si el sistema foucaultiano encuentra dificultades para nombrar un nosotros es porque los propios intereses de Foucault estribaban más en analizar el poder que la política. Al menos, en cuanto esta última tarea implica no solo la resistencia a dicho poder, sino también la creación de posibilidades alternativas de gobernarse. Siendo así, quizá lo más interesante sea completar la apuesta de Foucault con el pensamiento de otros autores. De lo contrario, corremos la tentación de transformar el cajón de herramientas foucaultiano en un corpus dogmático. Algo contra lo que, sin duda, el propio autor francés nos prevendría.

\section{BIBLIOGRAFÍA}

ARVIDSSON, Adam y COLLEONI, Elanor (2012). "Value in Informational Capitalism and on the Internet. The Information Society: An International Journal, 28 (3), pp. $135-150$.

BANDINELLI, Carolina y ARVIDSSON, Adam (2012). "Brand Yourself a Changemaker!". Journal of Macromarketing, 33 (1), pp. 67 - 71.

BENENTE, Mauro (2015). "Ideología y crítica en Michel Foucault. La cuestión del sujeto". Praxis Filosófica Nueva serie, 40, enero junio, pp. 183 - 206.

BOLTANSKI, Luc y CHIAPELLO, Éve (2002). El nuevo espíritu del capitalismo. Madrid: Akal.

\footnotetext{
5 Otra alternativa a esto, como señala Mario Domínguez, es trabajar por la desgubernamentalización de la subjetividad (Domínguez, 2018). No obstante, aún en ese caso seguiría siendo necesario plantear qué otros modos de subjetivación pueden plantearse como alternativas a la gubernamentalidad.
} 
BUTLER, Judith (2001a). "¿Qué es la crítica? Un ensayo sobre la virtud de Foucault": Instituto Europeo para Políticas Culturales Progresivas. Disponible en: http://eipcp.net/transversal/0806/butler/es [Consultado 05-11-2018].

- (2001b). Mecanismos psíquicos del poder. Teorías sobre la sujeción. Madrid: Ediciones Cátedra.

- (2007). El género en disputa. El feminismo y la subversión de la identidad. Barcelona: Paidós.

- (2009). Dar cuenta de sí mismo. Violencia ética y responsabilidad. Buenos Aires/Madrid: Amorrortu.

BROWN, Wendy (2015). El pueblo sin atributos. La secreta revolución del neoliberalismo. Barcelona: Malpaso Ediciones.

CADAHIA, Luciana y BLENGINO, Luis (2018). "Pueblo y heterogeneidad: claves para una gubernamentalidad popular". En VILLACAÑAS BERLANGA, José Luis y CASTRO ORELLANA, Rodrigo (eds.). Foucault y la historia de la filosofía. Madrid: Dado Ediciones, pp. 419 - 440.

CHIAPELLO, Ève (2003). "Reconciling the Two Principal Meanings of the Notion of Ideology The Example of the Concept of the 'Spirit of Capitalism'". European Journal of Social Theory, 6 (2), pp. $155-171$.

CONRAD MURRAY, Derek (2015). "Notes to self: the visual culture of selfies in the age of social media". Consumption Markets \& Culture, 18 (6), pp. 490 - 516.

CHOULIARAKI, Lilie (2010). "Self-mediation: new media and citizenship". Critical Discourse Studies, 7 (4), pp. 227 - 232.

DEBORD, Guy (1999). La sociedad del espectáculo. Valencia: Pre-textos.

DOMíNGUEZ SÁNCHEZ, Mario (2018). "Los usos políticos de Michel Foucault. Crítica de la filosofía política, liberalismo y ética". En VILLACAÑAS BERLANGA, José Luis y CASTRO ORELLANA, Rodrigo (eds.). Foucault y la historia de la filosofía. Madrid: Dado Ediciones, pp. $305-368$.

FEHER, Michel (2009). "Self-Appreciation; or The Aspirations of Human Capital". Public Culture, 21 (1), pp. $21-41$.

FOUCAULT, Michel (1994). “QQué es la ilustración? [Qu'est-ce que les Lumières?]”, Actual, 28, pp. 1 - 17.

- (1995). "¿Qué es la crítica? [Crítica y Aufklärung]", Daimon, Revista de Filosofía, 11 , pp. $5-25$.

- (1998). "El sujeto y el poder". Revista Mexicana de Sociología, 50 (3), pp. 3 - 20.

- (2009). Nacimiento de la biopolítica: curso en el Collège de France (1978-1979). Madrid: Akal.

FREEDEN, Michael (1996). Ideologies and Political Theory. A conceptual approach. New York: Oxford University Press. 
FUCHS, Christian (2010). "Labor in Informational Capitalism and on the Internet". The Information Society: An International Journal, 26, pp. 179 - 196.

— (2013). "With or Without Marx? With or Without Capitalism? A Rejoinder to Adam Arvidsson and Eleanor Colleoni", tripleC, 10(2), pp. 2012.

— and SANDOVAL, Marisol (2014). "Digital Workers of the World Unite! A Framework for Critically Theorising and Analysing Digital Labour", tripleC, 12(2), pp. 486 $-563$.

— and SEVIGNANI, Sebastian (2013). "What is Digital Labour? What is Digital Work? What's their Difference? And why do these Questions Matter for Understanding Social Media?", tripleC, 11(2), pp. 237 - 293.

HAN, Byung-Chul (2013). La sociedad de la transparencia. Barcelona: Herder.

HEARN, Alison (2008). "'Meat, Mask, Burden'. Probing the contours of the branded 'self'". Journal of Consumer Culture. 8 (2), pp. 163 - 183.

- (2017). "Verified: Self-presentation, identity management and selfhood in the age of big data". Popular Communication, 15 (2), pp. 62 - 77.

- y SCHOENHOFF, Stephanie (2016). "From Celebrity to Influence: Tracing the Diffusion of Celebrity Value across the Data Stream". En MARSHALL: David y REDMOND, Sean (eds.). A Companion to Celebrity. Chichester: John Wiley \& Sons, pp. $194-212$.

IQANI, Mehita y SCHROEDER, Jonathan E. (2016). "\#selfie: digital self-portraits as commodity form and consumption practice". Consumption Markets \& Culture, 19 (5), pp. $405-415$.

KHAMIS, Susie ANG, Lawrence y WELLING, Raymond (2017). "Self-branding, 'micro-celebrity' and the rise of Social Media Influencers". Celebrity Studies, 8 (2), pp. $191-208$.

KUEHN, Kathleen y CORRIGAN, Thomas F. (2013). "Hope Labor: The Role of Employment Prospects in Online Social Production. The Political Economy of Communication, 1(1), pp. $9-25$.

LAVAL, Christian. y DARDOT, Pierre (2013). La nueva razón del mundo. Ensayo sobre la sociedad neoliberal. Barcelona: Gedisa.

LAZZARATO, Maurizio (2013). La fábrica del hombre endeudado. Ensayo sobre la condición neoliberal. Buenos Aires/Madrid: Amorrortu.

LEMES DE CASTRO, Julio Cesar (2016). "Social networks as dispositives of neoliberal governmentality". Journal of Media Critiques, 2 (7), pp. 85 - 101.

LÓPEZ ÁLVAREZ, Pablo (2016). "Sigue cierta algarabía. Foucault, el neoliberalismo y nosotros". En CASTRO ORELLANA, Rodrigo. y SALINAS ARAYA, Adán (eds.). La actualidad de Michel Foucault. Madrid: Escolar y mayo, pp. 231 - 254.

MARWICK, Alice E. (2015). "Instafame: Luxury Selfies in the Attention Economy". Public Culture, 27 (1), pp. $137-159$. 
- (2016). "You May Know Me from Youtube. (Micro-)Celebrity in Social Media". En MARSHALL: David y REDMOND, Sean (eds.). A Companion to Celebrity. Chichester: John Wiley \& Sons, pp. $194-212$.

NAVARRO RUIZ, Clara (2015). "Efectos de subjetivación y 'sí mismo'. Modificaciones en la ontología crítica del presente de Michel Foucault". III Congreso "La actualidad de Michel Foucault". Madrid, 6-8 mayo 2015.

PAGE, Ruth (2012). "The linguistics of self-branding and micro-celebrity in Twitter: the role of hashtags". Discourse \& Communication, 6 (2), pp. $181-201$.

PETERS, Tom (1997). "The Brand Called You". Fast Company, 31 de agosto. Disponible en: https://www.fastcompany.com/28905/brand-called-you [Consultado 05-11-2018].

RANCIÈRE, Jacques (2011). "La política no es coextensiva ni a la vida ni al Estado", en RANCIÈRE, Jacques, El tiempo de la igualdad. Diálogos sobre política y estética. Barcelona: Herder.

READ, Jason. (2003). The Micro-Politics of Capital. Marx and the Prehistory of the Present. Albany: State University of New York Press.

RICOEUR, Paul (1986). Lectures on Ideology and Utopia, George E. Taylor (ed.). New York: Columbia University Press.

SENFT, Theresa (2013). "Microcelebrity and the branded self". En HARTLEY, John; BURGESS, Jean y BRUNS, Axel (eds.). A Companion to New Media Dynamics. Oxford: Wiley Blackwell.

SMITH, Adam (1958). Investigación sobre la naturaleza y causas de la riqueza de las naciones. México: Fondo de Cultura Económica.

SRNICEK, Nick (2017). Platform capitalism. Cambridge: Polity Press.

TIIDENBERG, Katrin y GÓMEZ CRUZ, Edgar (2015). "Selfies, Image and Re-making of the Body". Body and Society, 21 (4), pp. $1-26$.

TURNER, Graeme (2006). "The mass production of celebrity. 'Celetoids', reality TV and the 'demotic turn'". International journal of Cultural Studies, 9 (2), pp. 153 165.

WERNICK, Andrew (1991). Promotional culture. Advertising, ideology and symbolic expression. London: Sage Publications.

ZAJC, Melita (2015). "The Social Media Dispositive and Monetization of User-Generated Consent" The Information Society, 31 (1), pp. $61-67$.

ZAFRA, Remedios (2015). Ojos y capital. Bilbao: Consonni.

- (2017). El entusiasmo. Precariedad y trabajo creativo en la era digital. Barcelona: Anagrama.

ŽlŽEK, Slavoj (ed.). (1994). Mapping ideology, London/New York: Verso.

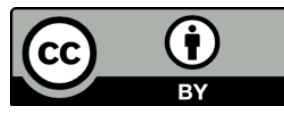

